\title{
Combining and optimizing NIRS and EEG to study inter- ictal epileptic discharges
}

\author{
Robert J Cooper ${ }^{\mathrm{a}}$, Meryem Yucel ${ }^{\mathrm{a}}$, Louis Gagnon ${ }^{\mathrm{a}, \mathrm{b}}$, Nao Suzuki ${ }^{\mathrm{a}}$, \\ Naoaki Tanaka ${ }^{\mathrm{a}}$, Claus Reinsberger ${ }^{\mathrm{a}, \mathrm{c}}$, David A. Boas ${ }^{\mathrm{a}}$ and Steve Stufflebeam ${ }^{\mathrm{a}}$ \\ ${ }^{a}$ Athinoula A. Martinos Center for Biomedical Imaging, Massachusetts General Hospital, Charlestown, MA, USA \\ ${ }^{b}$ Department of Electrical Engineering and Computer Science, MIT, Cambridge, MA, USA \\ 'Department of Neurology, Brigham and Women's Hospital, Boston, MA, USA
}

\begin{abstract}
We describe our ongoing application of NIRS-EEG to the study of inter-ictal discharges in adult epilepsy. We discuss optimizing NIRS-EEG data acquisition and analysis and we present preliminary NIRS-EEG results for an epileptic patient. OCIS codes: (170.0110), (170.1610)
\end{abstract}

\section{Introduction}

Epilepsy is a chronic neurological disorder characterized by spontaneous hyperactive and hyper-synchronous neuronal firing, often resulting in distinct clinical manifestations. In a sub-set of patients with drug-resistant partial epilepsy the regular occurrence of epileptic discharges can lead to a slow neurological and cognitive decline [1]. As a method of observing electro-cortical activity, EEG has been at the forefront of the study and diagnosis of epileptic conditions for decades. Although EEG contains a wealth of information, the changes in electrical potential at the scalp often do not accurately reflect the activity of cortical neurons [2]. Also, EEG provides little information about the effect of epileptic discharges on the health of surrounding brain tissues.

In the last decade, simultaneous EEG and BOLD-fMRI has emerged as an important tool in the study of epileptic conditions [3], though two fundamental limitations have yet to be overcome. First, the duration for which it is feasible to study an epileptic patient with EEG-fMRI is limited to 1-2 hours, making it difficult to study transient, spontaneous phenomena. Second, the BOLD signal (a measure of changes in deoxygenated hemoglobin concentration) is often difficult to interpret. The observation of negative BOLD signals is common in studies of epileptic discharges [4] and the true cause of these responses cannot be determined with BOLD alone.

Though the spatial coverage and resolution of NIRS cannot compete with that of fMRI, NIRS-EEG has several advantages over EEG-fMRI. NIRS provides a measurement of not only deoxyhemoglobin (HbR) concentration but also of oxyhemoglobin $(\mathrm{HbO})$ and, therefore, blood volume [5]. By estimating changes in blood flow using the Grubb relation [6], it is also possible to estimate localized changes in oxygen metabolism using NIRS. Couple this with a high temporal resolution and the fact that it can be applied for days at a time and it is clear that NIRS-EEG is a promising technique for studying the spatial, temporal, hemodynamic and metabolic characteristics of both ictal and inter-ictal epileptic events.

In this paper we describe a novel NIRS-EEG head cap and the application of this cap to the study of interictal epileptic discharges in our first patient. Essential to the development of this cap was the incorporation of several short separation $(\sim 10 \mathrm{~mm})$ NIRS channels which allow the effects of superficial hemodynamics to be regressed from the cortical signal using an adaptive filtering approach.

\section{Methods}

An array of optical fibers was fixed into a standard EEG head cap ('EasyCap', BrainVision LLC, USA) via a set of specialized ring adapters. These adapters allow the fibers to be locked into place in the cap and also hold an extra detector fiber $10 \mathrm{~mm}$ from each source fiber. The inclusion of such short-separation detectors has recently been shown to be of significant benefit to the processing of NIRS data by allowing superficial hemodynamic variations to be separated from those occurring in the cortex. The ring adapters allow access to the tip of the fiber so that hair can easily be moved and the rings can be fixed into the EEG cap in any position allowing the NIRS channels to cover any region of the head.

At present the NIRS-EEG cap holds 4 source fibers, 4 detector fibers, and 4 short-separation detector fibers (one for each source). This array covers an area of approximately $6 \times 10 \mathrm{~cm}$ and provides ten $30 \mathrm{~mm}$ NIRS channels and four $10 \mathrm{~mm}$ NIRS channels. The cap is designed in such a way that the 10-20 EEG electrode montage remains unaltered by the addition of the array of NIRS fibers. The size of the cap can be selected so as to best suit each patient and the position of the NIRS fibers in the cap can be chosen based on the MEG, EEG, fMRI and PET 


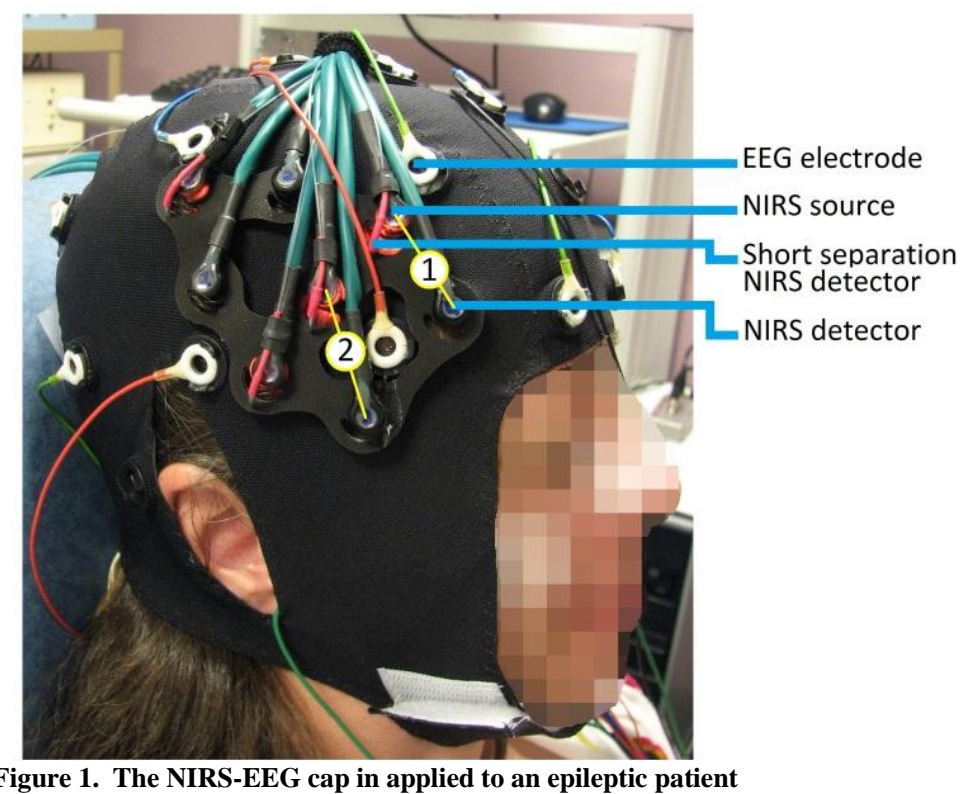

dd 3D Imaging OSA 2012

Figure 1. The NIRS-EEG cap in applied to an epileptic patient

recordings which are obtained as part of each epilepsy patient's clinical assessment. The cap is shown in position on an epileptic patient in figure 1 .

NIRS recording was performed using a CW6 system (TechEn Inc., USA) operating at 690 and $830 \mathrm{~nm}$. The EEG was recorded using a NeuroScan NuAmps system and sintered $\mathrm{Ag} / \mathrm{AgCl}$ ring electrodes from BrainVision LLC, USA. The two recordings were time-locked using an external timing signal. NIRS data were processed using a Kalman filtering approach within a general linear framework as described by Gagnon et al. 2011 [7]. This approach employs the short-separation NIRS signals as regressors in order to remove the superficial contribution to each normal-separation NIRS signal whilst simultaneously estimating the hemodynamic response function (HRF).

\section{Results}

A 36 year-old female with non-lesional, complex partial epilepsy and a high frequency of inter-ictal spiking underwent 1 hour of simultaneous NIRS-EEG. Written, informed consent was obtained in advance of the study. Previous MEG and EEG source localization results suggested that inter-ictal discharges were not well focused but were generally confined to the frontal, medial areas of the right temporal lobe. The NIRS probe was positioned over F8 and between the F4, T8 and C4 electrode positions so as to best sample this cortical area (see figure 1). The resulting EEG data was inspected by a clinical electrophysiologist (NT) and events identified as either 'epileptic' or 'suspicious'. Events defined as 'suspicious' were considered possibly epileptic though could not be definitely distinguished as such. This allowed us to produce a vector of event timings on which to base our linear model of the NIRS signal. During the 1 hour of recording, 43 epileptic and 37 suspicious discharges were observed. Figure 2 shows the inter-ictal HRF obtained from two NIRS channel for all events, both epileptic and suspicious. Fractional changes in concentration of hemoglobins and in estimated cerebral blood flow (rCBF) and the cerebral metabolic rate of oxygen $\left(\mathrm{rCMRO}_{2}\right)$ are provided. The values of $\mathrm{rCBF}$ and $\mathrm{rCMRO}_{2}$ were estimated using the methods described by Boas et al. [8]. The channel of figure 2a (labeled ' 1 ' in figure 1) shows a marked increase in $\mathrm{HbO}$, a smaller increase in $\mathrm{HbR}$ and a corresponding increase in both $\mathrm{rCBF}$ and $\mathrm{rCMRO}_{2}$ beginning after $\mathrm{t}=0$. Figure $2 \mathrm{~b}$ (channel '2' in figure 1) shows a distinct decrease in $\mathrm{HbO}, \mathrm{HbR}, \mathrm{rCBF}$ and $\mathrm{rCMRO}_{2}$.

\section{Discussion}

Our preliminary data clearly show that changes in hemoglobin concentrations, time-locked to inter-ictal epileptic discharges can be resolved using a NIRS-EEG approach. It is important to note that these changes are observed despite the fact that this patient's epileptic focus is deeper than can be directly sampled by NIRS. Our conclusion is therefore that these epileptic discharges caused significant changes in blood flow and metabolism in an extended volume of tissue. This is consistent with EEG-fMRI results, which indicate the affected volume varies markedly across subjects [9].

Interestingly, the changes in hemoglobin concentrations observed in this single patient do not follow the classical functional response profile (which consists of an increase in $\mathrm{HbO}$ and decrease in $\mathrm{HbR}$ ). Also, the observed response varies with position, with some NIRS channels measuring an increase in $\mathrm{HbO}$ and others a pronounced decrease. Patients with partial epilepsy often develop a region of continuous hypo-metabolism around 

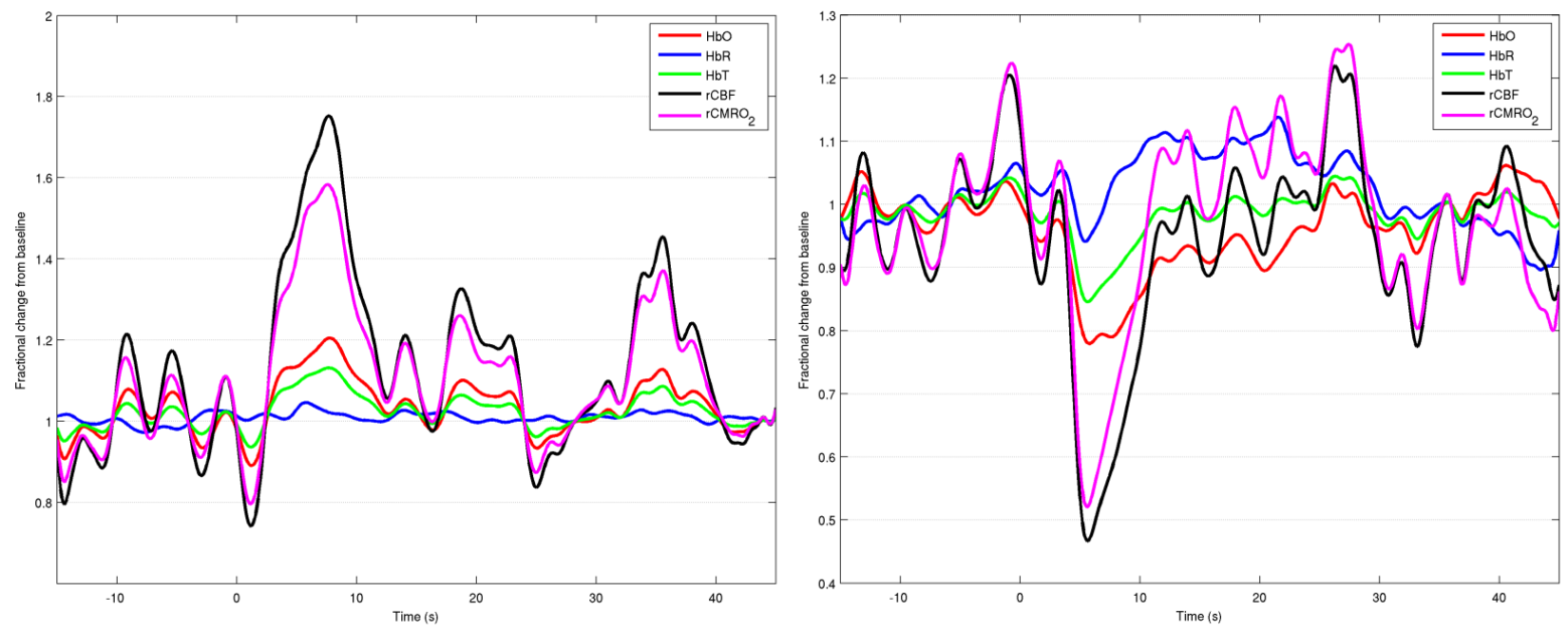

Figure 2. The hemodynamic and metabolic changes observed at two different NIRS channels in response to inter-ictal discharges

their epileptic focus, which is itself generally hypermetabolic [10]. It is possible that the variations in response observed in our data are a transient representation of this phenomenon.

It is clear from this data that NIRS-EEG can be used to successfully study inter-ictal epileptic events. The use of NIRS to calculate changes in $\mathrm{HbO}, \mathrm{HbR}$ and $\mathrm{CMRO}_{2}$ will provide a much clearer understanding of the impact of epileptic discharges than is commonly provided by EEG-fMRI. It will also allow a large amount of data to be obtained relatively easily, as NIRS-EEG can be applied continuously at the bedside. This is essential to the study of epileptic conditions because, as indicated by EEG-fMRI, results are highly variable both within and across subjects. In the data shown here, $\mathrm{CMRO}_{2}$ is merely an estimate because a measure of $\mathrm{CBF}$ is not available. However, we hope to extend our paradigm to enable simultaneous measures of blood flow using diffuse correlation spectroscopy (DCS). An arrangement capable of measuring $\mathrm{HbO}, \mathrm{HbR}$, blood volume and blood flow would provide an elegant and novel method for studying the impact of epileptic activity on brain tissues.

\section{Acknowledgements}

This work is funded by NIH grants P41-RR14075 and R01-EB006385. L.Gagnon is supported by the Fond Quebecois sur la Nature et les Technologies.

\section{References}

[1] A. Pitkänen and T. P. Sutula, "Is epilepsy a progressive disorder? Prospects for new therapeutic approaches in temporal-lobe epilepsy," The Lancet Neurology, vol. 1, no. 3, pp. 173-181, Jul. 2002.

[2] J. X. Tao, A. Ray, S. Hawes-Ebersole, and J. S. Ebersole, "Intracranial EEG substrates of scalp EEG interictal spikes," Epilepsia, vol. 46, no. 5, pp. 669-676, May 2005.

[3] A. Salek-Haddadi et al., "Hemodynamic correlates of epileptiform discharges: An EEG-fMRI study of 63 patients with focal epilepsy," Brain Research, vol. 1088, no. 1, pp. 148-166, May 2006.

[4] E. Kobayashi, A. P. Bagshaw, C. Grova, F. Dubeau, and J. Gotman, "Negative BOLD responses to epileptic spikes," Human Brain Mapping, vol. 27, no. 6, pp. 488-497, Jun. 2006.

[5] H. Obrig and A. Villringer, "Beyond the Visible - Imaging the Human Brain With Light," J Cereb Blood Flow Metab, vol. 23, no. 1, pp. 118, Jan. 2003.

[6] R. L. Grubb Jr, M. E. Raichle, J. O. Eichling, and M. M. Ter-Pogossian, "The effects of changes in PaCO2 on cerebral blood volume, blood flow, and vascular mean transit time,” Stroke; a Journal of Cerebral Circulation, vol. 5, no. 5, pp. 630-639, Oct. 1974.

[7] L. Gagnon, K. Perdue, D. N. Greve, D. Goldenholz, G. Kaskhedikar, and D. A. Boas, "Improved recovery of the hemodynamic response in diffuse optical imaging using short optode separations and state-space modeling," NeuroImage, vol. 56, no. 3, pp. 1362-1371, Jun. 2011.

[8] D. A. Boas et al., "Can the cerebral metabolic rate of oxygen be estimated with near-infrared spectroscopy?," Physics in Medicine and Biology, vol. 48, no. 15, pp. 2405-2418, Aug. 2003.

[9] C.-G. Bénar et al., "The BOLD Response to Interictal Epileptiform Discharges,” NeuroImage, vol. 17, no. 3, pp. 1182-1192, Nov. 2002.

[10] S. Dedeurwaerdere, B. Jupp, and T. J. O'Brien, "Positron Emission Tomography in Basic Epilepsy Research: A View of the Epileptic Brain,” Epilepsia, vol. 48, pp. 56-64, Sep. 2007. 